BOOK BRIEFLY NOTED

\section{Atlas of Anatomy}

A.M. Gilroy, B.R. MacPherson, and L.M. Ross, eds. New York: Thieme; 2008, 700 pages, 2200 illustrations, \$74.95.

his 700-page Atlas of Anatomy is richly illustrated with color drawings and multiple tables. It covers the entire body including the back, thorax, abdomen/pelvis, upper limb, lower limb, head and neck, and neuroanatomy. Although this atlas is not specifically written for neuroradiologists, there are valuable color plates showing and labeling multiple structures of the spine, base of the skull, and brain. This atlas may serve as a suitable anatomic text for a radiology residency library.

DOI 10.3174/ajnr.A1197 